\title{
Composite mesh migration into the sigmoid colon following ventral hernia repair
}

\author{
E. C. Nelson • T. J. Vidovszky
}

Received: 2 October 2009/Accepted: 22 December 2009/Published online: 8 January 2010

(C) The Author(s) 2010. This article is published with open access at Springerlink.com

\begin{abstract}
Ventral hernia repair is frequently performed with one of a number of mesh products, usually with good results. The current emphasis on laparoscopic repair has resulted in multiple composite mesh products for use in the peritoneal cavity. The addition of a second layer of more inert material to the mesh is intended to prevent adhesions with the underlying viscera, and multiple studies demonstrate effectiveness in doing this. Despite this, occasional complications may still present. We present the case of a 65 -year-old man coming to our clinic 5 years after a third ventral hernia repair/revision with composite mesh.
\end{abstract}

Keywords Composite mesh · Enterocutaneous fistula . Hernia $\cdot$ Mesh $\cdot$ Migration $\cdot$ Ventral hernia

\section{Introduction}

Ventral hernia repair is frequently performed with one of a number of mesh products, usually with good results. The current emphasis on laparoscopic repair has resulted in multiple composite mesh products for use in the peritoneal cavity. The addition of a second layer of more inert material to the mesh is intended to prevent adhesions with the underlying viscera, and multiple studies demonstrate effectiveness in doing this [1]. Despite this, occasional complications may still present [2]. We present the case of

E. C. Nelson $(\bowtie) \cdot$ T. J. Vidovszky

Department of Surgery, University of California,

Davis Medical Center, 2221 Stockton Blvd.,

Sacramento, CA 95817, USA

e-mail: enelson06m@gmail.com; enelson06m@yahoo.com

T. J. Vidovszky

e-mail: tamas.vidovszky@ucdmc.ucdavis.edu a 65-year-old man coming to our clinic 5 years after a third ventral hernia repair/revision with composite mesh.

\section{Case report}

In January 2009, a 65-year-old man presented to our surgery clinic complaining of copious yellow drainage from an infraumbilical midline abdominal wound consistent with an enterocutaneous fistula. His surgical history was significant for two ventral hernia repairs in 2001, the first for incarcerated hernia requiring bowel resection, and a ventral hernia repair/revision with a Bard Composix $4 \times 8$-in mesh in 2004. He was asymptomatic until September 2008, when he began to have abscesses in the area. These were initially treated with several incision and drainage procedures without improvement. By 2009, he required pad changes to the area every $2-6 \mathrm{~h}$ and had lost $40 \mathrm{lbs}$ due to the discomfort caused by the increased drainage associated with eating.

After an extensive preoperative workup necessary due to his complex cardiac history, he was taken to the operating room in February 2009. The findings included an ileocutaneous fistula, sigmoid colon mass, and abdominal wall abscess (Fig. 1). A small bowel resection of $20 \mathrm{~cm}$ of inflamed ileum with stapled functional end-to-end anastamosis was performed. Due to an incidentally discovered sigmoid mass with surrounding inflammation, a sigmoid colectomy was performed. Upon opening the specimen on the back table, retained mesh in the lumen of the colon was found (Fig. 2). Sutures from the prior repair were present in the abdominal wall associated with a large abscess. These were completely removed, along with necrotic portions of the abdominal wall, including the umbilicus. The abdominal wall was then reconstructed with component separation 


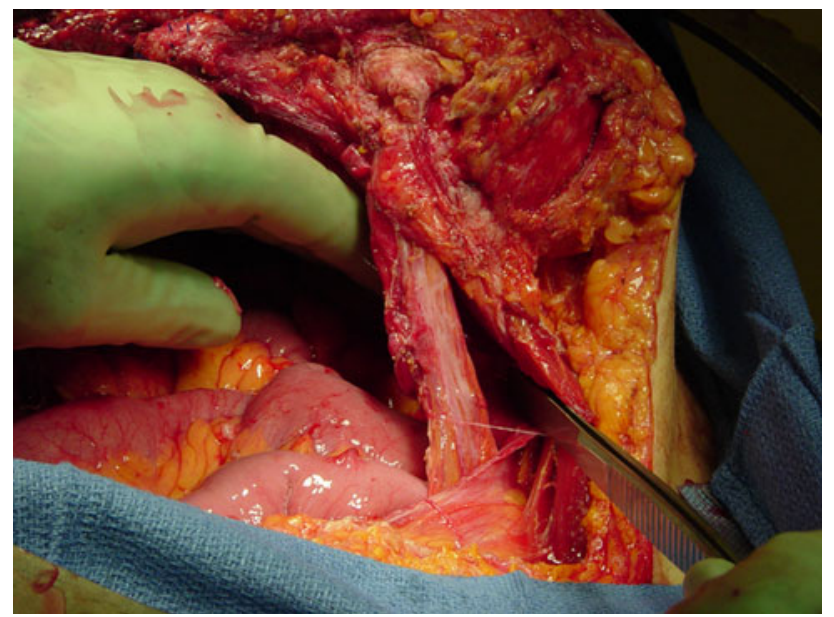

Fig. 1 Abdominal wall inflammatory mass with associated underlying enterocutaneous fistula

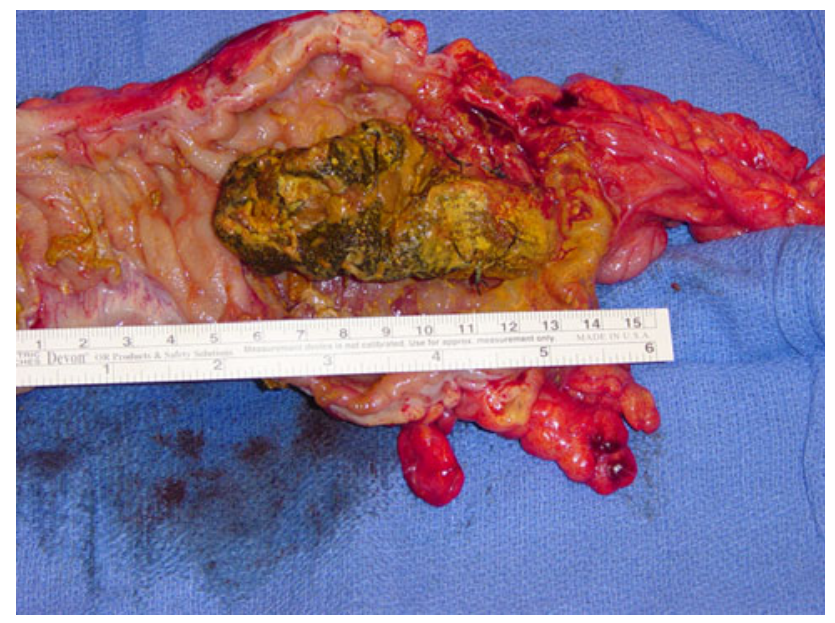

Fig. 2 Opened sigmoid colon with intraluminal mesh

and AlloDerm. The skin was only loosely approximated with staples.

After a somewhat prolonged postoperative course, the patient was discharged home. Due to the patient's social situation, no follow-up examinations have been possible. Phone calls to the patient confirm that his abdominal wound has completely closed and no recurrence of the enterocutaneous fistula has occurred. In addition, he has regained the lost weight.

\section{Discussion}

Ventral hernia repair is a common surgical problem, with about 500,000 cases diagnosed yearly in the US [3]. Though primary repair with component separation is an option, the majority of repairs are still performed with mesh. Composite mesh combines an inert surface with a more porous surface, allowing the intraperitoneal placement of mesh with minimal adhesion formation to the dorsal surface of the mesh, but tissue ingrowth to the ventral surface.

Serious complications of mesh repair should be uncommon [4]. Intraoperative complications due to bleeding or damage to the abdominal viscera may be avoided by meticulous technique. Postoperative infection should, likewise, be minimized by attention to standard guidelines. Other postoperative complications include herniation of the abdominal viscera around the edges of the mesh causing hernia recurrence and possible incarceration, migration, and erosion of the mesh. If the edges of the mesh are inadequately fixed to the abdominal wall, the ventral surface of the mesh may be exposed, thereby, increasing the risk of adhesions. Finally, damage to intraabdominal organs from defective mesh may be a concern with certain mesh products. The recent recall of certain Bard Composix mesh products was due to concerns regarding a "memory recoil ring" that might break, injuring nearby viscera [3].

One previous case of migration of a composite mesh with absorbable dorsal surface has been reported by Soler et al. [5]. The Dacron/Vicryl mesh created a small bowel fistula and migrated distally. To our knowledge, our case represents the first reported erosion and migration of permanent double-layer composite mesh used for a ventral hernia repair. We believe that the mesh first eroded into the small bowel near the site of the enterocutaneous fistula and then migrated intraluminally to the sigmoid colon. Possible contributing factors to this complication include inadequate fixation, inflammatory response due to the prior repairs, and unrecognized injury at surgery, though this final possibility is less likely.

\section{Conclusion}

Though composite mesh is generally safe for use intraabdominally, our case demonstrates that adhesions, erosion, and migration remain a concern. Possible predisposing factors include inadequate fixation and ongoing inflammation from prior surgeries.

Open Access This article is distributed under the terms of the Creative Commons Attribution Noncommercial License which permits any noncommercial use, distribution, and reproduction in any medium, provided the original author(s) and source are credited.

\section{References}

1. Gonzalez R, Rodeheaver GT, Moody DL, Foresman PA, Ramshaw BJ (2004) Resistance to adhesion formation: a comparative study of treated and untreated mesh products placed in the abdominal cavity. Hernia 8:213-219 
2. Hazebroek EJ, Leibman S, Smith GS (2009) Erosion of a composite PTFE/ePTFE mesh after hiatal hernia repair. Surg Laparosc Endosc Percutan Tech 19:175-177

3. Hope WW, Iannitti DA (2009) An algorithm for managing patients who have Composix Kugel ventral hernia mesh. Hernia 13:475-479

4. Amid P (2001) Complications of the use of prostheses: part 1. In: Bendavid R (ed) Abdominal wall hernias: principles and management. Springer-Verlag, New York, p 792
5. Soler M, Verhaeghe P, Essomba A, Sevestre H, Stoppa R (1993) Treatment of postoperative incisional hernias by a composite prosthesis (polyester-polyglactin 910). Clinical and experimental study. Ann Chir 47:598-608 